Bond University

Research Repository

\title{
:
}

\section{The Relationship Between Strength Measures and Task Performance in Specialist Tactical Police}

Orr, Rob Marc; Robinson, Jeremy ; Hasanki, Ksaniel ; Talaber, Kimberly; Schram, Ben; Roberts, Adam

Published in:

Journal of Strength and Conditioning Research

DOI:

10.1519/JSC.0000000000003511

Licence:

Other

Link to output in Bond University research repository.

Recommended citation(APA):

Orr, R. M., Robinson, J., Hasanki, K., Talaber, K., Schram, B., \& Roberts, A. (2022). The Relationship Between Strength Measures and Task Performance in Specialist Tactical Police. Journal of Strength and Conditioning Research, 36(3), 757-762. https://doi.org/10.1519/JSC.0000000000003511

\section{General rights}

Copyright and moral rights for the publications made accessible in the public portal are retained by the authors and/or other copyright owners and it is a condition of accessing publications that users recognise and abide by the legal requirements associated with these rights.

For more information, or if you believe that this document breaches copyright, please contact the Bond University research repository coordinator. 
Strength Measures and Task Performance 1

1 The Relationship Between Strength Measures and Task Performance in Specialist

Tactical Police 


\section{ABSTRACT}

4 Specialist Tactical Police Officers (STPO) carry heavier on-body loads than generalist police

5 officers. Improvements in strength may mitigate the impacts of these heavier loads. The aim

6 of this investigation was to determine the correlations between absolute and relative strength

7 measures and occupational task performance in STPOs. Retrospective data were provided for

847 male specialist police officers from an elite Australian police unit. Data included body mass

9 (mean $=89.0 \pm 8.58 \mathrm{~kg})$, strength measures (1 Repetition Maximum [RM] measures for a bench

10 press, squat, deadlift and pull-up) and task performance measures (85 kg victim drag wearing

$1115 \mathrm{~kg}$ of operational load and $5 \mathrm{~km}$ pack march wearing $40 \mathrm{~kg}$ of operational load). Pearson's

12 correlations were conducted to determine relationships between measures and were plotted on

13 a linear regressions model. Significant, moderate to strong correlations were found between all

14 strength measures and victim drag performance and significant negative moderate correlations

15 between relative bench press, absolute and relative squat and absolute and relative pull-up and

16 pack march times. The absolute deadlift had the strongest correlation to the victim drag

$17 \quad(r=0.747, p<0.01)$ while the relative pull-up showed the strongest correlation with pack march

18 performance $(r=-0.466, p<0.01)$. The requirement to lift a portion of the dummy off the ground

19 during the victim drag may explain the increased importance of absolute strength while the

20 requirement to transport load affixed to the body may explain the importance of relative

21 strength requirements. Improvements in absolute and relative upper and lower body strength

22 may improve task performance in this population.

23 Keywords: SWAT, Tactical Personnel, law enforcement, elite, load carriage 


\section{INTRODUCTION}

Police officers must be prepared to perform physically demanding and arduous occupational tasks that require them to run, jump, crawl, climb or push/pull with maximal exertion $(3,22$, 24). Furthermore, they may be required to perform these tasks while apprehending an uncooperative suspect $(28,29)$ and whilst carrying occupational loads (1). On average, these occupational loads can weight around $10 \mathrm{~kg}(1)$ and have been known to reduce officer power, agility, and change of direction speed $(13,19)$.

Policing situations which are deemed extreme in nature are handled by specialist tactical police officers such as those serving in Special Weapons and Tactics (SWAT) units (6). These specialist tactical units of the police forces consist of specialist tactical police officers (STPO) who are trained and employed to resolve critical incidents involving a threat to public safety (6). The critical nature of the tasks carried out by STPOs require them to carry threat-dependent weaponry, body armor, and equipment (such as breaching devices, gas masks, etc.) (6). This equipment can add an additional 10 to $30 \mathrm{~kg}$ of load to their bodies above that of general police officers $(1,5,14)$. With these necessary additions to the STPOs operational load, there is typically a concurrent detrimental effect on their ability to complete occupational tasks (5) and an increased physiological burden $(2,4)$. This increased physiological burden highlights the importance of developing and maintaining optimal physical fitness in $\operatorname{STPO}(7,8,23)$.

The requirement for STPO to be physically strong is noted in the literature, as is the fact that these officers are typically stronger than the general population (17). For example, previous research by Robinson et al. (25) found that both absolute and relative strength measures were associated with STPO load carriage performance over repeated $5 \mathrm{~km}$ load carriage events completed as fast as possible (pack weight of $25 \mathrm{~kg}$ ). Significant weak to strong correlations were found between strength measures of 1 Repetition Maximum (1RM) deadlift (absolute r=- 
0.288 ; relative $r=-0.403$ ), bench press (absolute $r=-0.360$ : relative $r=-0.465$ ), squat (absolute $\mathrm{r}=0.452$ : relative $\mathrm{r}=-0.500$ ), and pull-up (absolute $\mathrm{r}=0.452$ : relative $\mathrm{r}=0.607$ ) for the first march

with the later three measures significantly correlated with the subsequent second and third pack marches (each approximately 4 months apart). Of note however, across all four of these strength measures, the relative strength values were more strongly correlated to the load carriage task than the absolute values. Conversely, recent work by Moreno et al. (18) found that absolute deadlift measures were more strongly correlated to a 75 and $91 \mathrm{~kg}$ dummy drag than relative measures, with the latter not being significantly related. These findings suggest that while strength measures are important to the performance of tasks that can typically be undertaken by STPO, the nature of the strength, be it absolute or relative, may change depending on the task.

By understanding the different strength needs of STPO (in terms of relative or absolute strength) informed conditioning practices can be put in place to better optimize officer performance, especially in tasks where they may be weaker (e.g. load carriage versus a victim drag). The aim of this study was to investigate the relationship between measures of strength (absolute and relative) and task performance ( $85 \mathrm{~kg}$ victim drag and $5 \mathrm{~km}$ pack march) in STPO. It was hypothesized that strength would be related to performance on both tasks and that the nature of strength required (absolute and relative) would differ between tasks.

\section{METHODS}

\section{Experimental Approach to the Problem}

Retrospective data were provided from an elite Australian specialist police unit. Body mass data were provided in addition to data collected for strength performance (1RM bench press, 1RM squat, 1RM deadlift, 1RM pull-up) as part of departmental process. Relative strength data 
72 were derived by dividing 1RM scores by each individual's body mass. Furthermore, results

73 from two performance measures (85kg Victim Drag [15 kg officer load] and a 5km loaded [40

$74 \mathrm{~kg}$ officer load] Pack March) were also obtained as part of departmental processes.

\section{$75 \quad$ Subjects}

76 Data were obtained in non-identifiable format pertaining to 47 male STPOs from an

77 Australian law enforcement agency. The strict security protocols regarding the protective identity of these individuals limited all identifiable information to only body mass (mean $=$ $89.0 \pm 8.58 \mathrm{~kg}$ ) and the resulting performance. The limitation of demographic data in this population has been reported in previous literature (21). Ethics approval for this study were provided by the Bond University Human Research Ethics Committee (RO1585) and clearance to publish this information provided by the relevant law enforcement gatekeeper approvals.

\section{Procedures}

Strength measures were collected over two days (Day 1 - bench press and deadlift: Day 2 squat and pull-up) with the victim drag and pack march assessments conducted in the following week on independent days. Details regarding the protocols for the strength and performance measures are detailed below and has been previously described in the literature (25).

Strength Measures: The intent of a 1RM test is to determine the single maximal voluntary effort force that a muscle or muscle group can exert (11), and was utilized given its consideration as the gold standard for non-laboratory based strength assessments (10). The 1RM testing protocols were conducted as previously described by Haff and Triplett (11) and were always preceded by a 10-minute warm up on each day of testing. The warm up consisted of self-selected exercises such as Hindu pushups with rotation, bodyweight squats, clock lunges, supine gluteal bridges, 5-10kg medicine ball slams, push-ups and alternating lunges. 
96

97

98

99

100

101

102

103

104

105

106

107

108

109

110

111 The back squat utilized a 20kg Pendlay Barbell, Gym branded bumper weight plates in a

112 Hammer Strength Bench rack (LifeFitness, Rosemont, IL) with two S\&C coaches as spotters.

113 The subjects were instructed to position themselves so that the barbell was in contact with

114 upper fibers of the trapezius, above the scapulae. Foot and grip placements were instructed to

115 be slightly wider than the shoulders at a comfortable position. The test began with the officer

116 removing the barbell from the rack and taking two steps back to a pause. The participant was

117 instructed to perform the squat to $90^{\circ}$ of knee flexion before extending to full hip and knee

\section{The Bench Press}

Subjects completed the bench press testing using a 20kg Pendlay Barbell loaded with Gym branded bumper weight plates, utilizing a Hammer Strength Bench rack (LifeFitness, Rosemont, IL) and a Strength and Conditioning (S\&C) coach for safety. Subjects were instructed to lay supine on the bench with both feet flat on the floor with gluteals and scapulae in contact with the bench. Grip width was slightly wider than shoulder width (at a comfortable position) to ensure $90^{\circ}$ of elbow flexion was achieved at the end of the eccentric phase of the lift. The test initiated with the officer un-racking the weight and holding it with arms fully extended at the midline of their sternum. The barbell was lowered at a controlled speed until contact was made with the chest, and thereafter returned to the starting position above the sternum. A lift was only considered successful if the gluteals and scapulae remained in contact with the bench throughout the movement and the participant did not require any assistance from the spotter. The final load (including bar weight) lifted correctly was measured in $\mathrm{kg}$ to form the final score. extension. The final load (including bar weight) lifted correctly was measured in $\mathrm{kg}$ to form the final score. 


\section{The Deadlift}

121 The deadlift protocol was completed use an a 24kg diamond-shaped barbell (Australian Barbell

122 Company, Mordialloc, VIC) loaded with Gym branded bumper weight plates (LifeFitness,

123 Rosemont, IL). A rubber matted area of the gym was utilized for the testing. The subjects were

124 positioned inside the diamond shaped barbell and instructed to place their feet shoulder width 125 apart. The officers were instructed to squat, grip the barbell and maintain a neutral neck position 126 with feet flat on the ground. The test began with a cue followed by hip and knee extension in a

127 controlled manner. Once full hip and knee extension was achieved a second cue was provided 128 to lower the weight. Displays of poor lifting technique were met with cessation of the lift and 129 an unsuccessful lift result. The final load (including bar weight) lifted correctly was measured 130 in $\mathrm{kg}$ to form the final score.

\section{The 1 RM Pull-Up}

132 Subjects completed the pull-up assessment with a Dan Baker Strength weight belt 133 (DanBakerStrength Sunshine Coast, QLD, Australia), and Gym branded bumper plates 134 attached to hang in front of the body. The officers were instructed to grip the bar wider than 135 shoulder width at a comfortable position ensuring $90^{\circ}$ of elbow flexion at the end of the 136 concentric phase. The subjects were instructed to maintain a knee flexion at $\sim 90^{\circ}$ with ankles 137 crossed behind them during the movement. The test began on the with a cue to initiate the 138 concentric phase. A repetition was deemed successful if there was no swinging of the legs 139 during the movement and the chin was raised above parallel with the bar with $90^{\circ}$ of elbow 140 flexion. 1RM values achieved were the result of adding the officer's body mass to the external 141 weight lifted.

\section{Victim Drag protocol}


143 The victim drag was completed using an $85 \mathrm{~kg}$ Life Tec dummy placed with its head on the

144 starting line facing the direction of pull. A 50m long course was set up using Hart Sport cones

145 (Hart Sports, Brisbane, Australia) placed every 5m on a flat concrete surface. Subjects were

146 required to wear their tactical uniform, standard issue boots, body armour and helmet totalling

147 approximately $15 \mathrm{kgs}$ (loads varied slightly due to natural variations in individual clothing and

148 footwear sizes and subsequent ballistic plate sizes). The subjects were instructed to grip the

149 dummy under the arms and drag it backwards as fast and far as possible in the time allowed.

150 Each participant had $10 \mathrm{sec}$ to drag the dummy as far as possible before receiving a $20 \mathrm{sec}$ rest

151 where they could drop the dummy. This process was repeated 6 times, totalling $60 \mathrm{sec}$ of work

152 with $120 \mathrm{sec}$ of rest. The distance was scored by the number of markers passed by the feet of

153 the dummy by the end of the $6^{\text {th }}$ interval.

\section{Pack March protocol}

155 The $5 \mathrm{~km}$ pack march was completed over a course marked out on a combination of bitumen

156 and hard dirt surfaces. The subjects were required to wear their issued operational uniform,

157 boots, and body armour, alongside an unloaded primary weapon. On-body loads totalled 15

158 kgs which was measured with a Tanita BC82Fitplus scale (Tanita, Illinois, USA). In addition,

159 subjects wore an operational backpack which weighed $25 \mathrm{kgs}$ (Wedderburn Ds530 Digital

160 Industrial scale), leading to a total load of $40 \mathrm{~kg}$. The officers completed the $5 \mathrm{~km}$ march at

161 their own pace as fast as possible and time to completion was measured with a Hart Sports

162 hand held timer (Hart Sports, Brisbane, Australia). The final result was recorded in minutes

163 and seconds.

\section{Statistical Analyses}

165 Data were received in a non-identifiable format on Microsoft Excel spreadsheets and subsequently imported into a Statistical Package for the Social Sciences spreadsheet (Version 
167 23) for statistical analysis. A descriptive analysis to determine means and standard deviations

168 for body mass, independent variables (absolute and relative strength measures) and dependent

169 variables (Victim Drag and Pack March performance) was performed. A priori power analysis

170 was conducted using $\mathrm{G}^{*}$ Power software (Version 3.1.9.2, 2014) indicating a large effect size

$171(p=.5$, alpha $=0.05)$ could be detected with a 95\% confidence interval for the Pearson's

172 correlations given the population size. Pearson's correlations were performed on each measure

173 of strength (both absolute and relative) and both performance measures (Victim Drag and Pack

174 March). The strength of the correlations were defined as an $\mathrm{r}$ of between 0 to 0.19 as very weak;

1750.2 to 0.39 as weak, $40-.59$ as moderate, $60-.79$ as strong and .80-1.0 as very strong (9).

176 Correlations were plotted on a backward linear regressions model and analyzed for variance

177 (r2) between strength measures and occupational tasks. Alpha levels were set a 0.05 a priori.

\section{RESULTS}

179 The absolute and calculated relative results for each strength measure and the results of both

180 occupational tasks can be seen in Table 1. Table 2 shows the correlations between each strength measure and task performance. For the victim drag task, all absolute strength measures

182 displayed significant $(\mathrm{p}<.01)$ and strong correlations with drag performance while the relative results showed significant $(\mathrm{p}<.01)$ moderate correlations (Table 2). Of all strength measures, the absolute deadlift accounted for $56 \%$ of the variance in victim drag (Figure 1). For the pack march task, the relative strength measures for the bench press, squat, and pull-up displayed significant $(p<.05)$ weak to moderate $(p<0.01)$ correlations with the pack march results, while

187 only the absolute squat and pull-up showed significantly $(\mathrm{p}<.05)$ weak correlations with pack march performance. Of all the strength measures, the relative pull-up accounted for $22 \%$ of the variance in the pack march (Figure 2). 
***Insert table 2 here***

192

*** Figures 2 and 3 here***

193

\section{DISCUSSION}

194 The aim of this study was to investigate relationships between measures of strength and task performance in STPO. The results suggest that both absolute and relative strength are strongly correlated with victim drag results and absolute and relative squat and pull-up strength are

197 moderately correlated with pack march results. Strength, both absolute and relative, appears to 198 be important for STPO task performance. On this basis, the development and maintenance of these strength measures are an occupational requirement for STPO. Furthermore, these measures are of importance in return-to-work rehabilitation and reconditioning processes 201 following injury.

202 The interpretation of these results suggest that, while relative strength was correlated with

203 victim drag performance, absolute strength may be of greater importance. The findings of this study partially support the findings of Moreno et al (18). While Moreno et al. (18) $(n=30$ students) found no significant relationships between relative deadlift performance and a victim drag (75 \& $91 \mathrm{~kg}$ drag). they did find strong significant correlations between absolute deadlift 207 performance and a victim drag. Likewise, in a study measuring only absolute strength measures, Hendrickson et al. (12) found significant correlations between improvements in $1 \mathrm{RM}$ bench press $(r=0.32)$ and squat $(r=0.33)$ (the only strength values measured) and victim drag $(61.4 \mathrm{~kg})$ performance in a group of recreationally active civilian women $(\mathrm{n}=56)$, One

211 potential reason why absolute strength may be of greater importance in a victim drag, as 212 opposed to relative strength, lies in the requirement of the participant to lift a portion of the 213 dummy off the ground and drag this absolute load, which is not affixed to their body and remains extant regardless of the participant's body mass. 
215 Conversely, a pack march requires the participant to move a worn load as part of their body

216

217

218

219

220

221

222 mass, hence this becomes a relative load. Although this is known to increase energy costs (15), this may also mean that the relative strength of the carrier may be of greater importance. This supposition is supported by the findings in this study, whereby relative strength measures were more strongly correlated to pack march performance than absolute measures. In addition, the results of this study support the findings by Robinson et al. (25) who likewise found relative measures to be more strongly correlated to a pack march event than absolute measures.

In this study absolute pull-ups and squats were significantly, albeit weakly correlated to pack march performance while absolute bench press and deadlift were not. Conversely Robinson et al., (25) found all measures of an absolute bench press, squat, pull-ups and deadlift were moderately to weakly correlated to one pack march event; however, the deadlift was no longer significantly correlated to two later pack marches. Similarly, the study by Hendrickson et. al. (12) found no significant correlation between pack march performance $(3.2 \mathrm{k} \mathrm{m}$ with $32.7 \mathrm{~kg})$ and improvements in absolute bench press or squat.

The different findings to that of our own study, with respects to a loaded pack march performance, may be due to the different populations studied. There is an underlying cardiovascular demand for performance in a loaded pack march $(12,27)$ which would be expected to be present in tactical personnel who have been exposed to pack marching previously (26). The previous study used civilians who were instructed on how to perform these tasks, and therefore would likely have had little familiarity with conducting them.

The results of this study support research investigating optimal conditioning measures for pack marching. Systematic reviews by Orr, et al. (20) and Knapik, et al. (16) highlighted that pack marching can be improved by a combined resistance training and an aerobic training program. However, the results of this finding suggest that while resistance training and the development 
239 of strength is advised to improve load carriage performance, the development of relative

240 strength may provide of greater value for pack marching specifically.

242 A notable limitation to this study is the measures of strength and the occupational tasks

243 selected. These measures and tasks were those in use by the law enforcement agency and, as

244 the data provided were retrospective, were beyond the control of the researchers. However,

245 given that these measures are in use, this research informs those not only in this agency, but 246 other agencies who employ these assessments and tasks, on best means of optimizing 247 performance.

249 With the growing number of STPO units internationally, a greater need for research into training methods and optimizing performance has become increasingly evident $(7,29)$. The results of this study highlight the relationships between upper and lower limb strength (both absolute and relative) and the performance of two key tasks performed by STPO.

\section{PRACTICAL APPLICATIONS}

Strength and conditioning programs for candidates wishing to serve in, currently serving officers of, or injured officers returning to, specialist tactical police units should include the development of both absolute and relative strength, utilizing maximum strength training methods and movements for the upper and lower body as part of their strength / reconditioning program. Furthermore, if increased performance in a victim drag is desired increases in absolute strength may be of greater benefit given that the dummy represents an absolute load

261 of which a portion must be lifted from the ground and dragged. Conversely, during a pack 


\section{REFERENCES}

266

267

268

269

270

271

272

273

274

275

276

277

278

279

280

281

282

283

284

285

286

287

288

289

290

291

292

293

294

295

296

297

298

299

300

301

302

1. Baran K, Dulla J, Orr R, Dawes J, and Pope R. Duty loads carried by the LA sheriff's department deputies. Journal of Australian Strength \& Conditioning 26: 34-38, 2018.

2. Beekley MD, Alt J, Buckley CM, Duffey M, and Crowder TA. Effects of heavy load carriage during constant-speed, simulated, road marching. Mil Med 172: 592-595, 2007.

3. Blacker SD, Carter JM, Wilkinson DM, Richmond VL, Rayson MP, and Peattie M. Physiological responses of Police Officers during job simulations wearing chemical, biological, radiological and nuclear personal protective equipment. Ergonomics 56: 137-147, 2013.

4. Blacker SD, Fallowfield JL, Bilzon JLJ, and Willems MET. Physiological responses to load carriage during level and downhill treadmill walking. Medicina Sportiva 13: 116124, 2009.

5. Carlton SD, Carbone PD, Stierli M, and Orr RM. The Impact of Occupational Load Carriage on the Mobility of the Tactical Police Officer. Journal of Australian Strength \& Conditioning 22: 32-37, 2014.

6. Cartner R, Dugan K, Eells T, Hansen P, Kolman J, Watt R, Barker S, Gnagey J, Clark J, and Wood S. SWAT standards for law enforcement agencies. Presented at Proceedings of the National Tactical Officers Association, 2008.

7. Clark JG, Jackson MS, Schaefer PM, and Sharpe EG. Training SWAT teams: Implications for improving tactical units. Journal of Criminal Justice 28: 407-413, 2000.

8. Davis MR, Easter RL, Carlock JM, Weiss LW, Longo EA, Smith LM, Dawes JJ, and Schilling BK. Self-reported physical tasks and exercise training in Special Weapons and Tactics (SWAT) teams. Journal of Strength \& Conditioning Research 30: 32423248, 2016.

9. Evans JD. Straightforward statistics for the behavioral sciences. Brooks/Cole, 1996.

10. Fleck SJ and Kraemer W. Designing Resistance Training Programs, $4 E$. Human Kinetics, 2014.

11. Haff GG and Triplett NT. Essentials of strength training and conditioning 4th edition. Human kinetics, 2015.

12. Hendrickson NR, Sharp MA, Alemany JA, Walker LA, Harman EA, Spiering BA, Hatfield DL, Yamamoto LM, Maresh CM, Kraemer WJ, and NindI BC. Combined resistance and endurance training improves physical capacity and performance on tactical occupational tasks. Eur J Appl Physiol 109: 1197-1208, 2010.

13. Joseph A, Wiley A, Orr R, Schram B, and Dawes JJ. The Impact of Load Carriage on Measures of Power and Agility in Tactical Occupations: A Critical Review. International journal of environmental research and public health 15: 88, 2018. 
14. Keeler JM. The Effect of Tactical Tasks and Gear on Muscle Activation of SWAT Officers, in: College of Education. University of Kentucky, 2014.

15. Knapik J and Reynolds K. Chapter 11: Load carriage in military operations: a review of historical, physiological, biomechanical and medical aspects, in: Military Quantitative Physiology: Problems and Concepts in Military Operational Medicine. Maryland, USA: Borden Institute, 2012, pp 303-337.

16. Knapik JJ, Harman EA, Steelman RA, and Graham BS. A Systematic Review of the Effects of Physical Training on Load Carriage Performance. Journal of Strength \& Conditioning Research 26: 585-597, 2012.

17. Maupin D, Wills T, Orr R, and Schram BEN. Fitness Profiles in Elite Tactical Units: A Critical Review. International journal of exercise science 11: 1041-1062, 2018.

18. Moreno M, Dawes JJ, Balfany K, Orr RM, and Lockie R. Only the strong survive: Relationships between lower-body strength and power with the 75-kg and 91-kg body drag, in: National Strength and Conditioning Association National Conference and Exhibition. Washington D.C., USA, 2019.

19. Orr R, Kukić F, Cvorovic A, Koropanovski N, Janković R, Dawes J, and Lockie R. Associations between Fitness Measures and Change of Direction Speeds with and without Occupational Loads in Female Police Officers. International journal of environmental research and public health 16, 2019.

20. Orr R, Pope R, Johnston V, and Coyle J. Load carriage: Minimising soldier injuries through physical conditioning-A narrative review. Journal of Military and Veterans' Health 18: 31-38, 2010.

21. Orr R, Pope R, Peterson S, Hinton B, and Stierli M. Leg power as an indicator of risk of injury or illness in police recruits. International journal of environmental research and public health 13: 237, 2016.

22. Orr R, Pope R, Stierli M, and Hinton B. Grip strength and its relationship to police recruit task performance and injury risk: a retrospective cohort study. International journal of environmental research and public health 14: 941, 2017.

23. Pryor RR, Colburn D, Crill MT, Hostler DP, and Suyama J. Fitness characteristics of a suburban special weapons and tactics team. Journal of strength and conditioning research 26: 752-757, 2012.

24. Richmond VL, Rayson MP, Wilkinson DM, Carter JM, and Blacker SD. Physical demands of firefighter search and rescue in ambient environmental conditions. Ergonomics 51: 1023-1031, 2008.

25. Robinson J, Roberts A, Irving S, and Orr R. Aerobic Fitness is of Greater Importance Than Strength and Power in the Load Carriage Performance of Specialist Police. International journal of exercise science 11: 987-998, 2018.

26. Rudzki SJ. Weight-load marching as a method of conditioning Australian Army recruits. Mil Med 154: 201-205, 1989.

27. Silk A, Savage R, Larsen B, and Aisbett B. Identifying and characterising the physical demands for an Australian specialist policing unit. Applied Ergonomics 68: 197-203, 2018.

28. Sörensen L, Smolander J, Louhevaara V, Korhonen O, and Oja P. Physical activity, fitness and body composition of Finnish police officers: a 15-year follow-up study. Occupational Medicine 50: 3-10, 2000.

29. Williams JJ and Westall D. SWAT and non-SWAT police officers and the use of force. Journal of Criminal Justice 31: 469-474, 2003. 
Strength Measures and Task Performance 15

350 

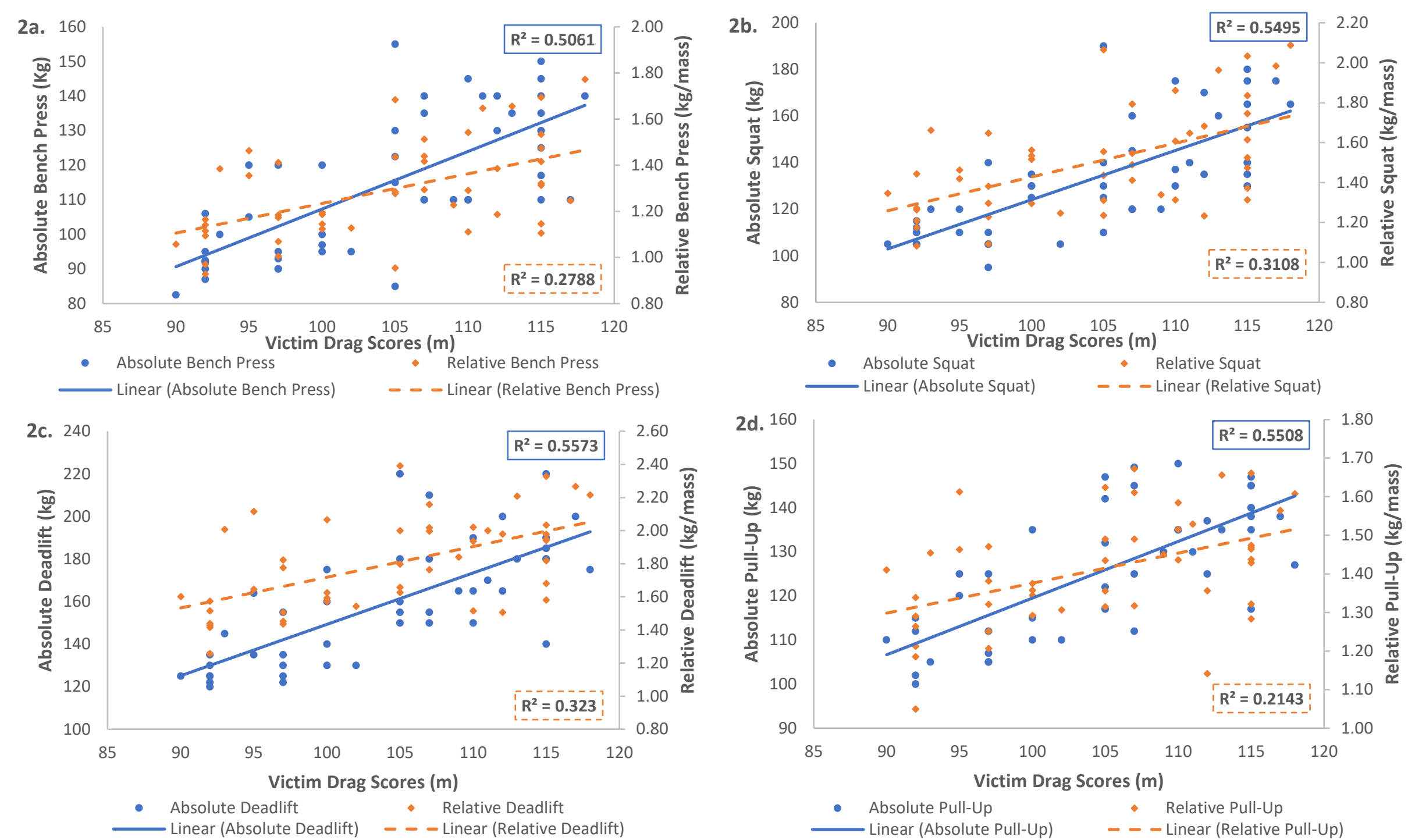

351 Figure 1. Linear regression analyses for absolute and relative strength measures and victim drag performance.

352 2a. Absolute/relative bench press correlation to victim drag performance, 2b. Absolute/relative squat correlation to victim drag performance,

353 2c. Absolute/relative deadlift correlation to victim drag performance, 2d. Absolute/relative pull-up correlation to victim drag performance. 

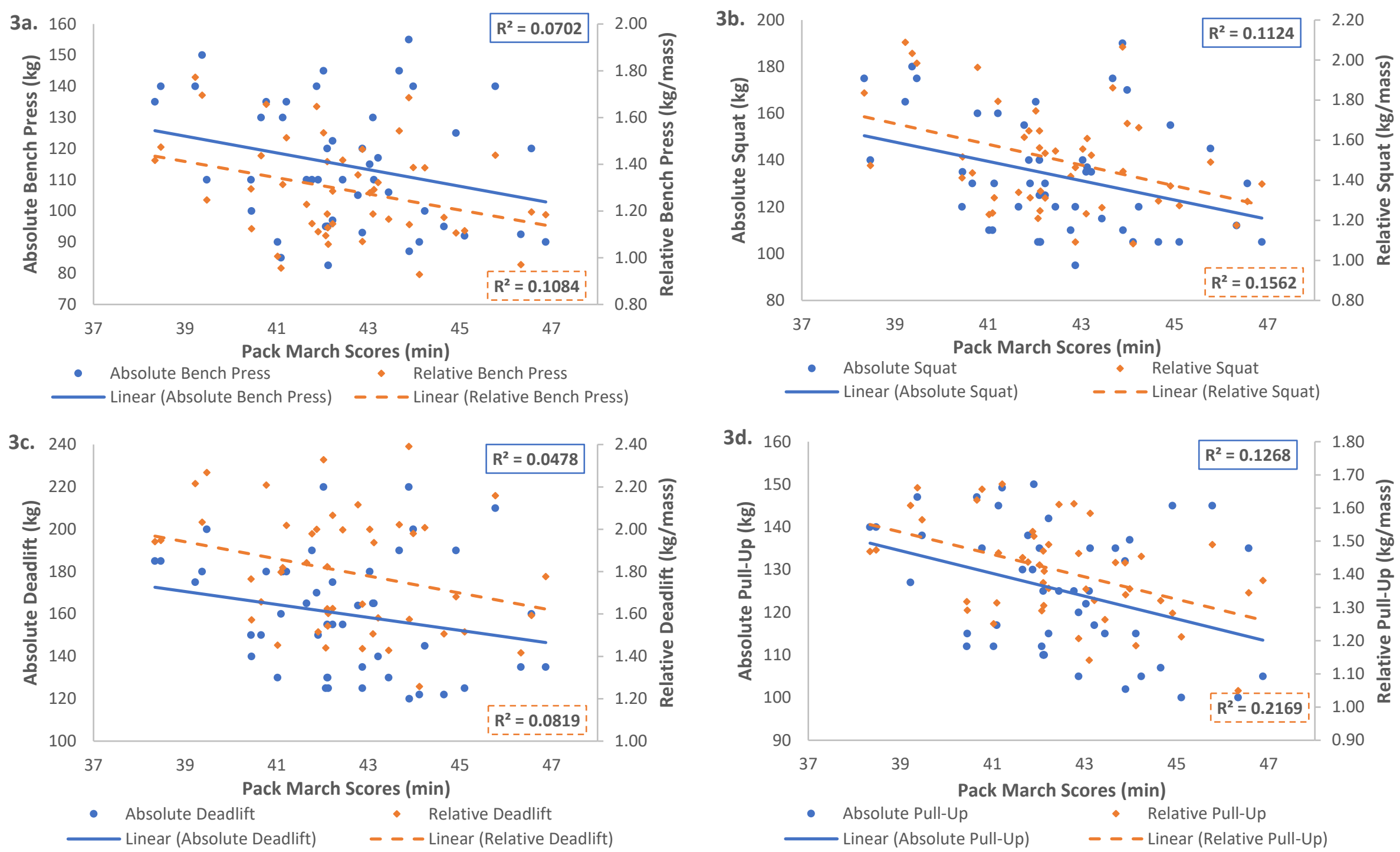

354 Figure 2. Linear regression analyses for absolute and relative strength measures and pack march performance.

355 3a. Absolute/relative bench press correlation to pack march performance, 3b. Absolute/relative squat correlation to pack march performance,

356 3c. Absolute/relative deadlift correlation to pack march performance, 3d. Absolute/relative pull-pp correlation to pack march performance. 


\section{TABLES}

358 Table 1. Mean results from all outcome measures and demographic data supplied.

\begin{tabular}{cc}
\hline Outcome Measure & Mean results \pm SD \\
\hline Body mass (kg) & $89.00 \pm 8.58$ \\
Absolute Bench (kg) & $114.68 \pm 20.15$ \\
Relative Bench & $1.29 \pm 0.21$ \\
Absolute Squat (kg) & $133.38 \pm 24.58$ \\
Relative Squat & $1.50 \pm 0.26$ \\
Absolute Deadlift (kg) & $159.96 \pm 27.88$ \\
Relative Deadlift & $1.80 \pm 0.28$ \\
Absolute Pull-up (kg) & $125.17 \pm 14.93$ \\
Relative Pull-up & $1.41 \pm 0.14$ \\
Victim Drag (m) & $104.40 \pm 8.61$ \\
Pack March (mins) & $42.48 \pm 1.99$ \\
\hline
\end{tabular}


361 Table 2. Correlation between outcome measures and performance in the victim drag and pack

362 march performance tests.

\begin{tabular}{ccc}
\hline Outcome Measure & Victim Drag & Pack March \\
\hline Absolute Bench & $r=0.711^{* *}$ & $r=-0.265$ \\
Relative Bench & $r=0.531 * *$ & $r=-0.330^{*}$ \\
Absolute Squat & $r=0.741^{* *}$ & $r=-0.335^{*}$ \\
Relative Squat & $r=0.557^{* *}$ & $r=-0.395 * *$ \\
Absolute Deadlift & $r=0.747 * *$ & $r=-0.219$ \\
Relative Deadlift & $r=0.568^{* *}$ & $r=-0.285$ \\
Absolute Pull-up & $r=0.742^{* *}$ & $r=-0.356^{*}$ \\
Relative Pull-up & $r=0.465^{* *}$ & $r=-0.468^{* *}$
\end{tabular}

363 Statistically significant at: $* \mathrm{p}<0.05 ; * * \mathrm{p}<0.01$ 
Strength Measures and Task Performance 0

364 